\title{
Transfer of incompatible spatial mapping to the vertical Simon task generalizes across effectors but not stimulus features
}

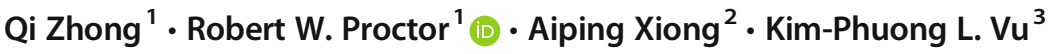 \\ Published online: 5 March 2020 \\ (C) The Psychonomic Society, Inc. 2020
}

\begin{abstract}
For the vertical Simon task, in which stimuli and responses are arrayed along the vertical dimension and stimulus location is irrelevant, a Simon effect (benefit for stimulus-response correspondence) is typically obtained. Results have been mixed about whether performing fewer than 100 trials of a spatially incompatible mapping prior to a Simon task reduces or eliminates this vertical Simon effect in a transfer session. Several reasons have been suggested to explain why previous studies show disparate results. Previously, we ruled out orientation of the response panel in the transverse or horizontal plane as a critical factor. The present experiments evaluated two other possible factors: finger/hand placement and relevant stimulus dimension. In Experiment 1, we found reduction of the vertical Simon effect for a circle-square discrimination after incompatible practice using a separate numeric keypad as the response device, regardless of whether the keypad was placed on a table and operated by index fingers or held in the hands and operated by thumbs. In Experiment 2, we replicated the reduction for the circle-square discrimination but found no evidence of reduction for a red-green color discrimination. Overall, our results suggest that the relevant discrimination of red-green color versus circle-square shape is responsible for the discrepancy in results across prior studies.
\end{abstract}

Keywords Simon effect $\cdot$ Spatial information processing $\cdot$ Stimulus-response compatibility $\cdot$ Visual choice reaction

\section{Introduction}

When people respond to the left and right locations of stimuli with left and right key-presses, they perform better when the mappings of stimuli and responses are spatially compatible (e.g., left response to left stimulus) than when they are not (e.g., left response to right stimulus). This spatial stimulusresponse compatibility (SRC) effect is evident in reaction time (RT) and error rate (Proctor \& Vu, 2006; Wang \& Proctor, 1996), and is quite robust. The spatial SRC effect also occurs when stimuli and response keys are arranged vertically, with top and bottom stimulus locations mapped to top and bottom response keys (Vu, Pellicano, \& Proctor, 2005; Vu \& Proctor, 2001).

Robert W. Proctor

rproctor@purdue.edu

1 Department of Psychological Sciences, Purdue University, West Lafayette, IN 47907, USA

2 College of Information Sciences and Technology, The Pennsylvania State University, E373 Westgate Building, University Park, PA 16802, USA

3 Department of Psychology, California State University Long Beach, 1250 Bellflower Blvd, Long Beach, CA 90840, USA
For tasks in which the stimulus locations are irrelevant and another stimulus dimension such as color is relevant, performance is better if the left or right location of the stimulus corresponds with the location of the response signaled by the relevant stimulus dimension than if it does not. This phenomenon is called the Simon effect (Simon, 1990; for a review see Lu \& Proctor, 1995). Simon (1969) hypothesized that the effect was caused by a tendency to react in the direction of the source of stimulation, although later accounts attribute the effect to spatial coding (Proctor \& Vu, 2006). The tendency to make the spatially congruent response is typically ascribed to long-term associations established through habit and experience (Tagliabue, Zorzi, Umiltà, \& Bassignani, 2000). However, numerous studies have now shown that the tendency to make the corresponding response can be reduced or reversed by prior practice with a spatially incompatible mapping (Proctor \& Lu, 1999; Tagliabue et al., 2000).

\section{Transfer of incompatible spatial mapping to a horizontal Simon task}

In Proctor and Lu's (1999) study, participants completed three sessions, each with 608 incompatible spatial-mapping practice trials, before performing the Simon task in a fourth, transfer 
session. In all sessions the stimulus and response locations were left and right. For the Simon task in the transfer session, RT was shorter on incongruent trials compared to congruent trials: The Simon effect was reversed to $-14 \mathrm{~ms}$. Vu (2007) employed 600 incompatible spatial-mapping practice trials prior to the Simon task in one of her experiments and found a reversed Simon effect of $-27 \mathrm{~ms}$. The results of these two studies indicate that performing a large number of trials with an incompatible spatial mapping reverses the direction of the horizontal Simon effect.

A smaller number of trials with the incompatible spatial mapping reduces the horizontal Simon effect, although the effect does not reverse (Tagliabue et al., 2000). For example, $\mathrm{Vu}$ (2007) conducted an experiment in which participants who performed 72 incompatible spatial mapping practice trials (plus eight warm-up trials) prior to the Simon task showed a nonsignificant Simon effect of $+4 \mathrm{~ms}$. Thus, these studies provide evidence that the difference between the congruent and incongruent conditions in the Simon task is reduced when preceded by less than 100 incompatible spatial-mapping practice trials and reversed to favor the incongruent relation when preceded by 600 or more of those trials.

\section{Transfer of incompatible spatial mapping to a vertical Simon task}

The Simon effect is also obtained when the stimuli and responses differ along vertical dimensions, although there has been some debate as to whether the underlying basis of this vertical Simon effect is the same as that for the horizontal Simon effect (e.g., Conde, Fraga Filho, Lameira, Riggio, \& Gawryszewski, 2017; Stürmer, Leuthold, Soetens, Schröter, \& Sommer, 2002; Töbel, Hübner, \& Stürmer, 2014). Often, responses are made on keys placed on the table top (i.e., the transverse plane), for which the response keys are not only in top (or upper) and bottom (or lower) locations in the plane but also far and near locations relative to the participant. However, because standard keyboard locations are referred to as upper and lower, and the Simon effect is similar in size to that obtained when the response keys are mounted in the frontal plane, researchers often refer to the key locations in vertical terms (e.g., Stürmer et al., 2002; Töbel et al., 2014). We maintain that description and terminology here.

Researchers have also used vertical stimulus-response arrangements to examine the influence of prior practice with an incompatible spatial mapping on the Simon effect using response devices arrayed in the frontal or transverse plane (Conde et al., 2015; Vu, 2007; Zhong, Xiong, Vu, \& Proctor, 2018). Vu (2007) found that, for responding on the transverse plane, following 600 practice trials with a spatially incompatible mapping, the vertical Simon effect was -1 ms. However, with only 80 trials of practice with an incompatible spatial mapping, a substantial vertical
Simon effect of $25 \mathrm{~ms}$ was still evident. When responses were made by using the index fingers to pull the levers of keys (microswitches) mounted in the frontal plane, Conde et al. (2015) also obtained a reduction of the vertical Simon effect following practice with an incompatible mapping (nonsignificant $+3 \mathrm{~ms}$ ), but in this case with only 100 trials. Conde et al. (2015) concluded that the difference in amount of incompatible practice needed to reduce the vertical Simon effect was due to the coding methods of the arrangement of response keys and arrangement of stimuli. They argued that because stimuli were presented in up and down locations on the screen, if the response keys were also located up and down in the frontal plane, people would code both stimuli and responses as up and down. However, when response keys were located on the transverse plane of the tabletop, as in Vu's study, people would code response keys as far and near rather than up and down.

To test Conde et al.'s (2015) hypothesis, Zhong et al. (2018) used a numeric keypad, operated by the thumbs, that was held vertically (so that the response keys were top and bottom in the frontal plane) or horizontally (so that the response keys were aligned in the transverse plane). When stimuli were presented in top and bottom locations on the screen, no matter whether the response keys were located on the transverse or frontal plane, Simon effects were reduced to a nonsignificant level after 100 trials of incompatible spatial practice. Even when the response planes of practice trials and Simon task trials differed (e.g., the practice trials involved responses on the transverse plane, but the Simon test trials involved responses on the frontal plane), the Simon effect in the transfer session was still reduced to a non-significant level. Thus, Zhong et al.'s results are generally in agreement with those of Conde et al. (2015), but provide evidence counter to the hypothesis that the orientation of the response device was responsible for the difference between Vu's (2007) and Conde et al.'s (2015) results.

\section{Current study}

Zhong et al. (2018) found significant transfer after 100 practice trials with an incompatible spatial mapping on the vertical Simon effect when the response keys were arranged along the transverse plane, similar to Vu's (2007) study in which a sizeable Simon effect was still evident. Because the orientation of the response device was the same in both studies, orientation can be excluded as the cause of the apparent discrepancy between the transfer effects. Comparing the two studies, we identified three other possibly critical factors. The first is that the placements of the fingers and hands differed. $\mathrm{Vu}$ (2007) had participants use their index fingers to respond on the number pad of a QWERTY keyboard placed on a table top, whereas Zhong et al. (2018) had participants hold a numeric 
keypad with their hands and respond with their thumbs. Hand posture and thumb versus index finger affect the speed and accuracy of data entry performance on mobile devices (Wobbrock, Myers, \& Aung, 2008), and response coding in choice reaction tasks has been shown to depend on hand posture in some situations (Cho \& Proctor, 2002).

Second, the types of number pads used in the two studies were different. $\mathrm{Vu}$ (2007) used the number pad at the right side of a QWERTY computer keyboard, whereas Zhong et al. (2018) used a separate, cordless numeric keypad. Distinct hand postures are required to press the keys with these two response apparatuses because of the additional keys on the one attached to the QWERTY keyboard. Third, the relevant stimulus dimensions differed. Vu (2007) used red and green circles as stimuli, whereas Zhong et al. (2018) used square and circle outline shapes as stimuli. Because the Simon effect often varies across the RT distribution (Proctor, Miles, \& Baroni, 2011), if the overall RTs to the color and shape stimuli differ, different patterns for the Simon effect could be generated indirectly. The present study sought to ascertain whether any of these factors is crucial for the incompatible practice to transfer to the vertical Simon task.

Experiments $1 \mathrm{~A}$ and $1 \mathrm{~B}$ aimed to determine whether the hand and finger placements cause distinct patterns of transfer effects, with the Simon task requiring the circlesquare discrimination. Participants responded on a horizontal numeric keypad that was either placed on the table and operated with the index fingers or held in the hands and operated with the thumbs. Experiments $2 \mathrm{~A}$ and $2 \mathrm{~B}$ compared the method used by $\mathrm{Vu}$ (2007), for which red and green stimuli are responded to with the index fingers on the number pad of a QWERTY keyboard placed on a table, with that for circle and square stimuli using the same mode of responding. Comparison of this latter condition to the results of Experiment 1, in which responses were made on a separate numeric keypad, provided evidence as to whether any factor associated with the different apparatuses plays a meaningful role. We also report RT distribution analyses to evaluate whether the time course of the Simon effect differs across stimulus conditions.

\section{Experiments 1A and 1B: Simon task for relevant stimulus shapes with and without prior incompatible spatial practice}

Experiment 1 was performed in two parts. Part A was conducted to obtain a baseline vertical Simon effect for a form discrimination task without prior practice of a task for which stimulus location was relevant. In Part B, participants performed a task in which they responded with an incompatible spatial mapping to stimuli in top and bottom locations, and then they were transferred to the vertical Simon task. In both Experiment $1 \mathrm{~A}$ and Experiment 1B, for the Simon task, participants were to respond to circle and square stimuli with presses of upper and lower keys on a numeric keypad. They used one of two finger/hand placements throughout the experiment: (a) hands prone, with index fingers on vertically aligned keys of the pad, which lay on a tabletop; (b) hands supine, with thumbs on vertically aligned keys of the pad, which was held in the hands parallel to the tabletop. The main goal was to determine whether the amount of reduction of the Simon effect after 100 trials of incompatible practice was less with the table/index finger placement (as in $\mathrm{Vu}, 2007$ ) than with the hand/thumb placement (as in Zhong et al., 2018).

\section{Method}

Participants Undergraduate students enrolled in Introductory Psychology courses at Purdue University took part for credits toward a course requirement. Ninety-six participants (43 male; 53 female), mean age 19.2 years $(S D=1.23)$ were included in Experiment 1A, and another 96 in Experiment 1B (42 male; 54 female), mean age 19.3 years $(S D=1.36)$. An additional five participants were omitted from each experiment, all for exceeding a $10 \%$ error criterion except one in Experiment $1 \mathrm{~B}$ for using incorrect fingers. All participants had normal or corrected-to-normal vision. In Experiment 1A, 84 were right-handed, four were left-handed, and eight were ambidextrous, whereas in Experiment 1B the corresponding numbers were 83 , seven, and six, respectively. All participants were naïve to the experiment's purpose.

Stimuli and apparatus The stimuli were similar to those used by Conde et al. (2015). For the incompatible spatial task, performed initially by participants in Experiment 1B, the stimuli were black solid circles of $0.5^{\circ}$ diameter located $6.5^{\circ}$ below or above a fixation point. For the Simon task, which was performed by all participants in both experiments, the stimuli were black outlines of a circle and a square. The square was $1^{\circ} \times 1^{\circ}$, and the circle was a diameter of $1^{\circ}$, presented above or below the fixation point at a distance of $6.5^{\circ}$ from center of the stimulus to center of the screen. The fixation point was a black cross. All stimuli were displayed on a white background. Participants sat in front of the monitor at a distance of approximately $57 \mathrm{~cm}$.

The experiments were conducted with a Dell Optiplex 745 personal computer with a Dell 19-in. LCD color monitor. Stimulus presentation, response recording, and feedback were controlled by E-prime 2.0 software. Responses were made on the " 2 " and " 8 " keys, the lower and upper positions of a vertical row on a Logitech Cordless 
Number Pad. Half of the participants held the numeric keypad flat in their hands, parallel to the tabletop, and pressed the keys with the thumbs. For the other half, the numeric keypad was on the tabletop, and responses were made with the index fingers.

Procedure The procedure used in the present study was approved by Purdue University's Institutional Review Board. After signing consent forms, participants performed the experiment in a dimly lit, quiet room. Each participant sat directly in front of the display screen and was instructed to respond to the stimulus using the numeric keypad. In each experiment, half (48) of the participants were told to hold the keypad horizontally with their thumbs on keys " 2 " or " 8 " (the upper and lower number keys in the center column of the $3 \times 3$ number array). The other half (48) were told to position their index fingers on the same response keys of the numeric keypad, which was placed on the tabletop. Within each condition, 24 participants used their left-hand fingers (thumbs or index fingers) to press the lower " 2 " key and their right-hand fingers to press the upper " 8 " key, whereas for the other 24 participants this relation was reversed. The experimenter remained in the room during the experiment to ensure that participants maintained the instructed posture.

Each trial started with onset of the fixation point for $1,500 \mathrm{~ms}$, after which a $400-\mathrm{Hz}$ warning tone was presented for $100 \mathrm{~ms}$. Then, a stimulus was presented above or below the fixation point and was shown until a response was recorded. In Experiment 1B, for the practice task, participants were told to respond to the location of the solid circle based on a spatially incompatible mapping (press the " 2 " key for circle above fixation and the " 8 " key for circle below fixation). They performed the incompatible spatial task for 100 trials, of which the first 20 trials were considered as warm-up and not included in the data analysis. After a 5-min break in Experiment 1B, and without the prior incompatible practice in Experiment 1A, participants performed the Simon task.

For the Simon task, participants were told to respond to the displayed circle or square by pressing the " 2 " or " 8 " key. The circle was assigned to one response and the square to the other response, with the assignment counterbalanced across participants. Each participant performed 100 trials of the Simon task, the first 20 of which were excluded from the data analysis as practice, as in the studies of Conde et al. (2015) and Zhong et al. (2018). There was an equal number of congruent and incongruent trials. For congruent trials, the stimulus was presented on the same side as the correct response, whereas for incongruent trials, the stimulus was on the opposite side of the correct response. Participants were told to keep their thumbs or index fingers on the response keys during the whole experiment and to respond as quickly and accurately as possible. Participants in Experiment 1B used the same finger/hand placement for the Simon task as for the incompatible spatial task.

\section{Results}

Spatial-mapping practice task of Experiment 1B Trials with error responses (3.9\%) were excluded from the RT analysis, as were outlier trials with RT $<100 \mathrm{~ms}$ or $>1,000 \mathrm{~ms}(1.1 \%)$. A one-way analysis of variance (ANOVA) with the betweensubject factor of finger/hand placement was applied to RT and error rate (ER). The effect of finger/hand placement was not significant on RT, $F<1.0$ (406 ms when the numeric keypad was held in the hands; $419 \mathrm{~ms}$ when the keypad was on the tabletop), or on error rate, $F<1.0$ (error rate of .037 when the numeric keypad was held in hands compared to .041 when it was placed on the tabletop).

Simon task of Experiments $1 \mathrm{~A}$ and 1B Trials with error responses $(2.5 \%$ in Experiment $1 \mathrm{~A}$ and $3.2 \%$ in Experiment 1B) were excluded from the RT analysis, as were outlier trials with RT $<100 \mathrm{~ms}$ or $>1,000 \mathrm{~ms}(0.8 \%$ in Experiment $1 \mathrm{~A}$ and $1.5 \%$ in Experiment 1B). ANOVAs for both RT and ER had three factors, 2 (congruency: congruent vs. incongruent) $\times 2$ (prior practice: practice vs. no-practice) $\times 2$ (finger/hand placement: numeric keypad held in hands vs. placed on tabletop). The first factor was within-subjects and the last two were between-subjects. Where appropriate, Greenhouse-Geissercorrected probabilities are reported but with the original degrees of freedom. Effects were regarded as statistically significant if $p<.05$. Mean RTs and ERs are provided in Table 1.

Reaction time Congruency had a significant main effect, $F_{(1,188)}=135.65, p<.001, \eta_{p}^{2}=.419$. Mean RT was $21 \mathrm{~ms}$ shorter for the congruent condition (461 ms) than for the incongruent condition (482 ms). The main effect of prior practice was not significant, $F<1.0$. Without the prior spatially incompatible practice, RT was $469 \mathrm{~ms}$, whereas with the spatially incompatible practice, RT was $473 \mathrm{~ms}$. The finger/hand placement condition also did not have a significant main effect, $F<1.0$. Mean RT was $471 \mathrm{~ms}$ regardless of whether responses were with thumbs on the hand-held numeric keypad or with index fingers on the pad placed on the tabletop.

The two-way interaction of congruency and finger/hand placement was nonsignificant, as was that of prior practice and finger/hand placement, $F s<1.0$. Of most importance, there was an interaction of congruency $\times$ prior practice, $F_{(1,188)}=22.19, p<.001, \eta_{p}^{2}=.106$, but no three-way interaction of those variables with finger/hand placement, $F<1.0$. The Simon effect was $30 \mathrm{~ms}$ for each finger/hand placement 
Table 1. Mean reaction time and error rate in the Simon task as a function of experiment, condition, and congruency

\begin{tabular}{|c|c|c|c|c|c|}
\hline \multirow[b]{2}{*}{ Experiment } & \multirow[b]{2}{*}{ Condition } & \multicolumn{2}{|c|}{ Reaction time (ms) } & \multicolumn{2}{|c|}{ Error rate $(\%)$} \\
\hline & & Congruent & Incongruent & Congruent & Incongruent \\
\hline \multicolumn{6}{|l|}{$1 \mathrm{~A}$} \\
\hline \multirow[t]{2}{*}{ W/O Practice } & Index fingers & $455(8.8)$ & $485(9.3)$ & $1.6(.3)$ & $3.7(.5)$ \\
\hline & Thumbs & $453(7.3)$ & $483(7.6)$ & $1.1(.3)$ & $3.6(.5)$ \\
\hline \multicolumn{6}{|l|}{ 1B } \\
\hline \multirow[t]{2}{*}{ With Practice } & Index fingers & $465(9.0)$ & $478(8.9)$ & $3.1(.4)$ & $2.9(.5)$ \\
\hline & Thumbs & $468(9.4)$ & $480(9.1)$ & $3.1(.5)$ & $4.1(.5)$ \\
\hline \multicolumn{6}{|l|}{$2 \mathrm{~A}$} \\
\hline \multirow[t]{2}{*}{ Color } & $\mathrm{W} / \mathrm{O}$ practice & $452(11.0)$ & $470(10.7)$ & $2.1(.4)$ & $3.6(.5)$ \\
\hline & With practice & $439(11.3)$ & $454(10.6)$ & $2.5(.6)$ & $3.8(.6)$ \\
\hline \multicolumn{6}{|l|}{$2 \mathrm{~B}$} \\
\hline \multirow[t]{2}{*}{ Shape } & W/O practice & $440(9.7)$ & $464(9.8)$ & $3.4(.7)$ & $4.7(.6)$ \\
\hline & With practice & $473(8.8)$ & $480(10.1)$ & $4.4(.9)$ & $3.9(.5)$ \\
\hline
\end{tabular}

without the spatially incompatible prior practice but only $12 \mathrm{~ms}$ for the tabletop placement and $13 \mathrm{~ms}$ for the handheld placement after such practice. Separate ANOVAs on the RT data from Experiments $1 \mathrm{~A}$ and $1 \mathrm{~B}$ showed that the Simon effect was significant both prior to and after practice, $F s_{(1,94)}=186.81$ and $18.74, p s<.001, \eta_{p}^{2}=.665$ and .166 .

To evaluate the dynamics of the Simon effect, an additional ANOVA was performed on the effect with RT bin as a factor. For each participant, RTs for congruent and incongruent trials were rank ordered from shortest to longest and split at the 20th, 40th, 60th, and 80th percentiles into five bins. The difference between incongruent and congruent trials was computed for each bin, and these Simon effect data were analyzed (see Fig. 1). The only $F$ ratio involving bin to exceed 1.0 was the nonsignificant main effect, $F_{(4,752)}=3.09, p=.063, \eta_{p}^{2}=$ .016: As evident in the figure, there was only a slight tendency for the Simon effect to decrease from Bins 2 to 5 .

Error rate Congruency showed a significant main effect on $\mathrm{ER}, F_{(1,188)}=21.45, p<.001, \eta_{p}^{2}=.102$. ER was smaller for the congruent condition (.022) than for the incongruent condition (.036). Finger/hand placement did not show any effect, $F<1.0$, with ER being .030 when responding with thumbs on the numeric keypad held in hands and .028 when responding with index fingers on the keypad placed on the tabletop. However, prior practice showed a main effect, $F_{(1,188)}=$ $5.07, p=.025, \eta_{p}^{2}=.026$ : ER was higher after spatially incompatible practice (.033) than without the prior practice $(.025)$.

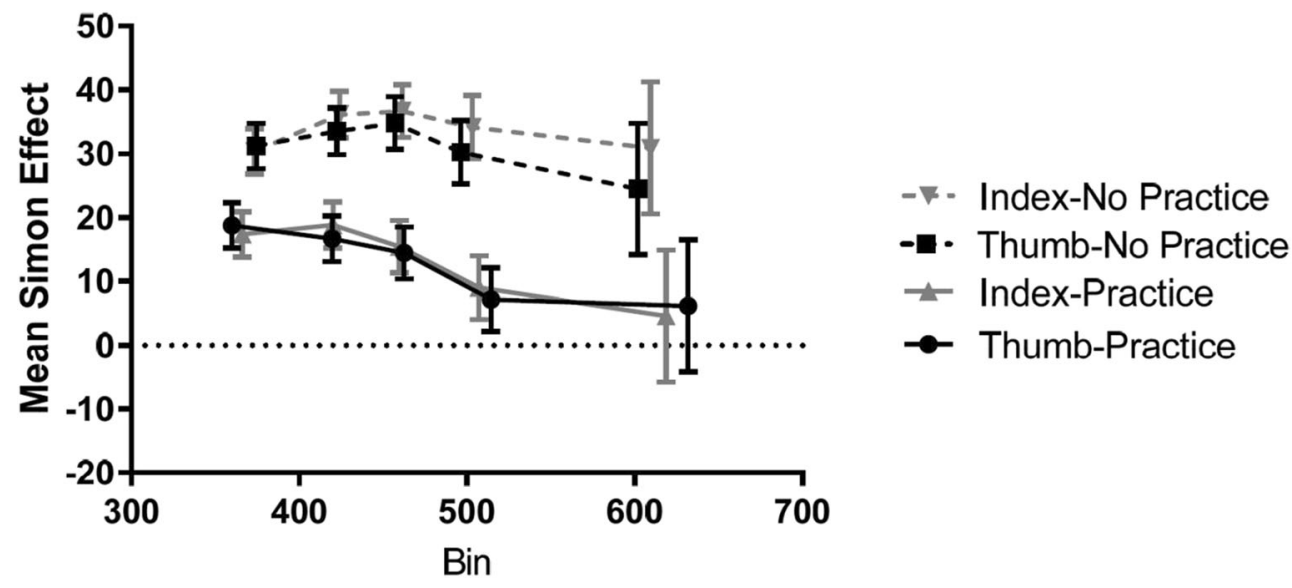

Fig. 1. Mean Simon effect in milliseconds for the reaction time bins as a function of whether responses were with the index fingers or thumbs and whether the Simon task was preceded by prior practice with an incompatible spatial mapping. Error bars represent \pm 1 SEM 
As with RT, the interaction between congruency and finger/ hand placement was not significant, $F_{(1,188)}=1.87, p=.173$, $\eta_{p}^{2}=.010$, nor was that of prior practice and finger/hand placement, $F_{(1,188)}=1.67, p=.198, \eta_{p}^{2}=.009$. The interaction between congruency and prior practice was significant, though, $F_{(1,188)}=10.38, p=.002, \eta_{p}^{2}=.052$, but the threeway interaction of those variables with finger/hand placement was not, $F<1.0$. After the spatially incompatible practice, the Simon effect was reduced from .023 to .004. Separate ANOVAs for Experiments $1 \mathrm{~A}$ and $1 \mathrm{~B}$ showed the Simon effect to be significant without the prior practice, $F_{(1,94)}=$ $32.59, p<.001, \eta_{p}^{2}=.257$, but not after the incompatible spatial practice, $F<1.0$.

\section{Discussion}

The results of Experiment 1 showed transfer of the incompatible spatial practice to the Simon task that did not differ significantly for the two finger/hand placements. The Simon effect was reduced by the prior spatially incompatible practice, but the effect was still significant in the RT data. That this pattern did not interact with finger/hand placement is evidence that transfer of the association between incongruent stimulus and response locations depended little if any on the placement. Furthermore, the RT distribution analysis showed only a slight tendency in the means for the Simon effect to decrease as RT increased, and no interaction of bin with the other variables. The lack of interaction of bin with the effect of prior incompatible practice is in agreement with findings reported by Conde et al. (2017) using the response device of Conde et al. (2015). Because any effect of the prior incompatible practice in reducing the Simon effect was similar across all bins for both finger/hand placements of the present experiment, finger/hand placement can be excluded as the factor that caused the apparent difference between transfer patterns for the vertical Simon effect in Vu's (2007) and Conde et al.'s (2015) studies.

\section{Experiments 2A and 2B: Simon task for color and shape discriminations with and without prior incompatible spatial practice}

Experiment 1 provided evidence that finger/hand placement was not the cause of the apparent difference in transfer effects of spatially incompatible practice on the vertical Simon task. Experiment 2 tested another hypothesis, whether the relevant stimulus feature influences transfer of the spatially incompatible practice to the Simon task. The design of Experiment 2A was similar to Vu's (2007) Experiment 3 in that red and green circles were used as the stimuli in the Simon task. To make Experiment 2A comparable to Vu's experiment, responses were made on the number pad of a QWERTY keyboard, as in her study.
Experiment 2B used outlines of square and circle stimuli in the Simon task, as in Zhong et al.'s (2018) research and Experiment 1 of the present study, with the same method of responding. Thus, the only difference between Experiment 2A and Experiment 2B was the stimuli used for the Simon task.

\section{Method}

Participants A total of 96 undergraduate students from the same subject pool as in Experiment 1 participated in Experiment 2. Of the 48 students in Experiment 2A, 28 were male and 20 were female, with a mean age of 19.48 years $(S D$ $=1.22$ ). A total of 40 of them were right-handed, three were left-handed, and five participants were ambidextrous. Of the 48 students in Experiment 2B, 33 were male and 15 were female, with a mean age of 19.25 years $(S D=1.52)$. Of these, 40 were right-handed, four were left-handed, and four were ambidextrous. Six additional participants were excluded from each experiment for exceeding the $10 \%$ error criterion.

Stimuli, apparatus, and procedure The same computer and software were used for recording the data. Instead of the separate numeric keypad, the number pad of a QWERTY keyboard was used. The stimulus display was made more comparable to that of $\mathrm{Vu}$ (2007). The screen was black, and the stimuli for the practice task were white circles of approximately $1^{\circ}$ of visual angle. In Experiment $2 \mathrm{~A}$ the stimuli for the Simon task were red or green solid circles of the same size, and in Experiment $2 \mathrm{~B}$ the stimuli were white square and circle forms of the same size as those used in Experiment 1. The response keys were " 8 " and " 2 " on the keyboard's number pad (located to the right side), and all participants placed their left index finger on the " 8 " and right index finger on the " 2 ." With this placement, the fingers of the left hand rested on a region of the keyboard without keys and at least the two rightmost fingers of the right hand were off of the keyboard. Within each experiment, 24 participants performed both the spatial incompatible practice and the standard Simon task; the other 24 performed only the standard Simon task.

For the practice session, participants were given the spatially incompatible mapping, as in Experiment 1B. For the Simon task session, participants in Experiment 2A were told to respond to the colors of the red and green circles, whereas those in Experiment 2B were told to respond to the shapes of the stimuli. Mappings of stimuli to responses were counterbalanced across participants, as in Experiment 1. In other ways, the method was like that of Experiment 1.

\section{Results}

Incompatible spatial practice session Half of the participants in each sub-experiment performed a practice session with a spatially incompatible mapping. A total of $4.0 \%$ of trials for 
which the response was incorrect and $1.6 \%$ of trials for which RT was $<100 \mathrm{~ms}$ or $>1,000 \mathrm{~ms}$ were excluded from the practice session of Experiment 2A. The corresponding percentages for Experiment $2 \mathrm{~B}$ were $3.5 \%$ and $1.4 \%$. For Experiment 2A, mean RT of the spatially incompatible practice trials was $426 \mathrm{~ms}$, and ER was .040. For Experiment 2B, RT of the spatially incompatible practice trials was $403 \mathrm{~ms}$, and ER was of .035 . Neither of these differences between experiments was significant: for RT, $F_{(1,46)}=1.13, p=.294$; for ER, $F<1.0$.

Simon task A total of $3.9 \%$ of trials for which the response was incorrect and $0.8 \%$ of trials for which RT was $<100 \mathrm{~ms}$ or $>1,000 \mathrm{~ms}$ were excluded from analysis. ANOVAs for RT and ER had three factors, 2 (congruency: congruent vs. incongruent $) \times 2$ (prior practice: practice vs. no-practice $) \times 2$ (stimulus feature: color vs. shape), with the first factor being within-subjects and the last two being between-subjects. Mean RTs and ERs are provided in Table 1.

Reaction time Congruency had a main effect on RT, $F_{(1,92)}=$ $52.14, p<.001, \eta_{p}^{2}=.362$. The difference between the congruent $(451 \mathrm{~ms})$ and incongruent $(470 \mathrm{~ms})$ conditions was 19 ms. Neither prior incompatible practice, $F<1.0$, nor stimulus feature, $F_{(1,92)}=1.15, p=.286, \eta_{p}^{2}=.012$, had a significant main effect, with mean RT of $457 \mathrm{~ms}$ for the color stimuli and $464 \mathrm{~ms}$ for the shape stimuli.

The interaction of congruency $\times$ stimulus feature was not significant, $F<1.0$. That between stimulus feature and practice also was not significant, $F_{(1,92)}=3.74, p=.056, \eta_{p}^{2}=.039$. However, individual analyses conducted for those participants who did not receive prior practice and those who did showed the following. Without the prior incompatible practice, RT did not differ significantly for the shape and color discriminations ( $M \mathrm{~s}=452$ and $461 \mathrm{~ms}$, respectively), $t_{(94)}=0.83, p=.411$. After incompatible practice, RT was longer for the shape discrimination than for the color discrimination $(M \mathrm{~s}=477 \mathrm{~ms}$ and $446 \mathrm{~ms}$, respectively), $t_{(94)}=2.96, p=.004$. Most important, the interaction between congruency and prior practice was significant, $F_{(1,92)}=5.25, p=.024, \eta_{p}^{2}=.054$. The Simon effect was reduced from $21 \mathrm{~ms}$ to $11 \mathrm{~ms}$ by spatially incompatible practice. Both the Simon effect without the prior practice, $F_{(1,46)}=44.19, p<.001, \eta_{p}^{2}=.490$, and with the practice, $F_{(1,46)}=12.45, p=.001, \eta_{p}^{2}=.213$, were significant. The three-way interaction with stimulus feature was not significant, $F_{(1,92)}=2.09, p=.151, \eta_{p}^{2}=.022$, providing indeterminate evidence as to whether the influence of the prior incompatible practice differed across the color and shape stimuli.

Because our concern was with the specific result patterns produced by the two stimulus sets, we analyzed the data of Experiments $2 \mathrm{~A}$ and $2 \mathrm{~B}$ separately. For Experiment 2B, in which the stimuli were squares and circles, there was a significant interaction between congruency and prior practice, $F_{(1,46)}=10.76, p=.002, \eta_{p}^{2}=.190$. The Simon effect for the condition without the prior practice was $24 \mathrm{~ms}$ (440 ms for congruent and $464 \mathrm{~ms}$ for incongruent) and statistically significant, $F_{(1,23)}=39.49, p<.001, \eta_{p}^{2}=.632$, whereas that for the condition with the prior practice was only $7 \mathrm{~ms}$ (473 ms for congruent and $480 \mathrm{~ms}$ for incongruent) but still significant, $F_{(1,23)}=4.93, p=.037, \eta_{p}^{2}=.176$. These results replicate closely those of Experiment 1B. In contrast, for Experiment $2 \mathrm{~A}$, in which the stimuli were red and green circles, there was no interaction between congruency and prior practice, $F<1.0$. The Simon effect without the prior practice was $18 \mathrm{~ms}$ (452 ms for congruent and $470 \mathrm{~ms}$ for incongruent) and statistically significant, $F_{(1,23)}=12.92, p=0.02, \eta_{p}^{2}=.360$, and the Simon effect with the prior practice was $15 \mathrm{~ms}$ (439 ms for congruent and $454 \mathrm{~ms}$ for incongruent), which was also significant, $F_{(1,23)}=7.67, p=.011, \eta_{p}^{2}=.250$.

Because $\mathrm{Vu}$ (2007) excluded only the first 12 trials as warm-up, and the trials that are closer in time to the practice task should be impacted the most, we conducted a supplementary analysis excluding only the first 12 trials. With the additional eight trials included for the Simon task, the three-way interaction was significant, $F_{(1,92)}=6.22, p=.014, \eta_{p}^{2}=.063$. The Simon effect for color stimuli was $13 \mathrm{~ms}$ without prior incompatible practice and $17 \mathrm{~ms}$ after incompatible practice, whereas that for the shape stimuli was $25 \mathrm{~ms}$ without practice and $6 \mathrm{~ms}$ after practice. These supplementary analyses, though not preplanned, provide converging evidence that the impact of the incompatible practice was more for the task requiring shape discriminations than for the task requiring color discriminations.

Finally, we conducted a bin analysis on the Simon effect similar to that performed for Experiment 1 (see Fig. 2). The data were noisier than in that experiment, in this case showing no bin main effect, $F_{(4,368)}=1.46, p=.237, \eta_{p}^{2}=.016$, but also no significant interactions of bin with the other factors: $\mathrm{Bin} \times$ Stimulus, $F<1.0 ;$ Bin $\times$ Practice, $F_{(4,368)}=2.06, p=.143, \eta_{p}^{2}$ $=.022 ;$ Bin $\times$ Stimulus $\times$ Practice, $F_{(4,368)}=1.36, p=.256, \eta_{p}^{2}$ $=.015$. Thus, there again was no statistically reliable evidence beyond an overall influence of the prior incompatible practice on mean RT.

Error rate Congruency showed a main effect, $F_{(1,92)}=8.35, p$ $=.005, \eta_{p}^{2}=.083$. ER for the congruent condition (.031) was smaller than that for the incongruent condition (.040). Prior practice did not show a main effect on ER, $F<1.0$, but stimulus feature did, $F_{(1,92)}=4.34, p=.040, \eta_{p}^{2}=.045$. ER was lower when responding to the color stimuli (.030) than when responding to the shape stimuli (.041). 


\section{Experiment 2}

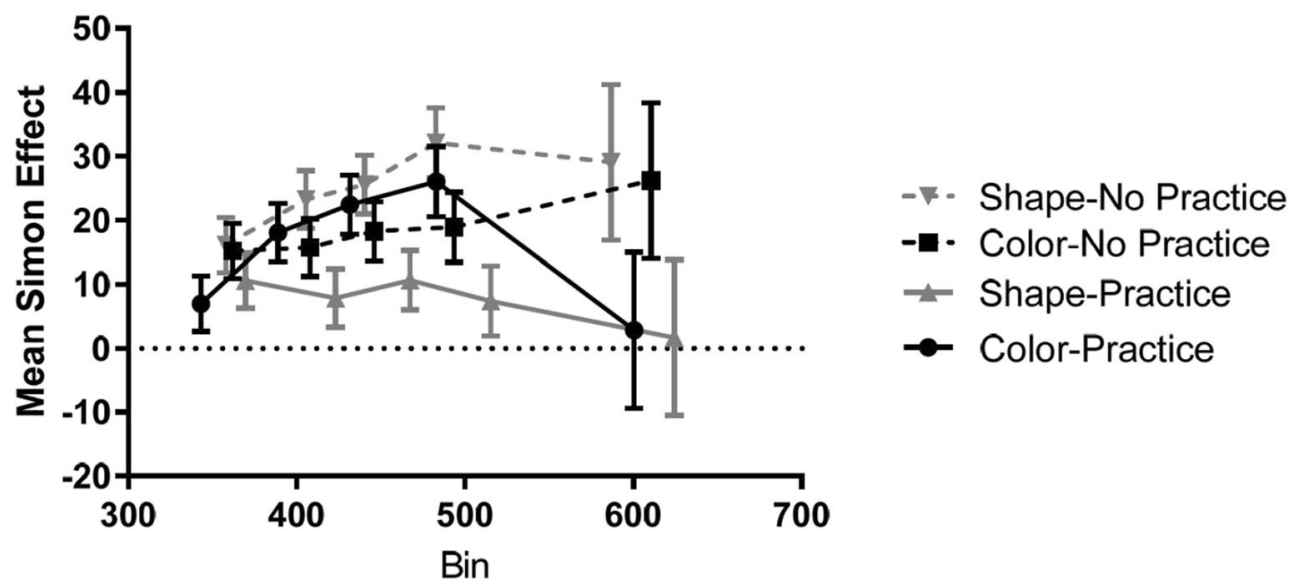

Fig. 2. Mean Simon effect in milliseconds for the reaction time bins as a function of whether the Simon task required shape or color discriminations and whether the Simon task was preceded by prior practice with an incompatible spatial mapping. Error bars represent \pm 1 SEM

For the ER data, the interactions of congruency $\times$ stimulus feature, $F_{(1,92)}=2.61, p=.110, \eta_{p}^{2}=.028$, prior practice $\times$ stimulus feature, $F<1.0$, and prior practice $\times$ stimulus feature $\times$ congruency, $F_{(1,92)}=1.63, p=.206, \eta_{p}^{2}=.017$, were not significant. The congruency $\times$ prior practice interaction also was not significant, $F_{(1,92)}=2.89, p=.093, \eta_{p}^{2}=.030$. However, separate analyses revealed that the Simon effect of ER was significant without prior practice, $F_{(1,92)}=18.04, p<$ $0.01, \eta_{p}^{2}=.282$, but not after practice with the incompatible spatial mapping, $F<1.0$. Separate analyses of the ER data of Experiments $2 \mathrm{~A}$ and $2 \mathrm{~B}$ similarly showed no significant interaction of congruency $\times$ prior practice, but with the Simon effect reduced from significant to nonsignificant by spatially incompatible practice.

\section{Discussion}

In Experiment 2, an overall reduction of the vertical Simon effect for RT was found. For the color stimuli (Experiment 2A), the 3-ms reduction in the Simon effect after the prior practice was not significant, and the 15-ms Simon effect after the incompatible spatial practice was significant. For the shape stimuli (Experiment 2B), the Simon effect was reduced by the spatially incompatible practice, but the 7-ms effect after practice still was significant. Although the three-way interaction in the overall ANOVA was nonsignificant, the supplementary analysis excluding fewer trials as practice provided additional evidence that the color stimuli showed little influence of the incompatible practice on the vertical Simon effect compared to the shape stimuli.

A bin analysis was conducted to determine whether the magnitude of the Simon effect across the RT distribution indirectly contributed to the differences in mean Simon effects for the color and shape stimuli. This analysis showed no significant bin effect or interaction of bin with the other variables, meaning that any differential influence of the type of discrimination or prior incompatible practice across the distributions was not strong enough to be statistically reliable.

A reduction of the vertical Simon effect in ER for both stimulus types was found as well. Even though no significant transfer effect of the spatially incompatible practice on ER was observed in the ANOVAs, the vertical Simon effect was significant without prior practice and not significant after practice in both stimulus conditions.

\section{General discussion}

A significant vertical Simon effect was found in Experiments 1A, 2A, and 2B when the Simon task was performed without prior practice of the incompatible spatial task. These results are consistent with each other and also replicate the results of previous studies (e.g., Conde et al., 2015; Vu, 2007; Zhong et al., 2018). The vertical Simon effect was significant no matter whether participants responded with their index fingers or thumbs, to red and green circles or outlines of squares and circles, and on a number pad of a QWERTY keyboard or a cordless keypad.

In both Experiment 1 and Experiment 2, prior practice with the incompatible spatial mapping significantly reduced the Simon effect for the task requiring circle-square discriminations, although a small effect remained. This result pattern also generalized across different response effectors and keypads. Combined with the findings of Zhong et al. (2018), we can conclude that 100 trials of practice with an incompatible spatial mapping is sufficient to reduce the vertical Simon effect for circle-square shape stimuli, replicating the findings of Conde et al. $(2015,2017)$. 
For the red-green color stimuli (Experiment 2), there was no significant reduction in Simon effect size due to the prior practice with 100 trials of the incompatible spatial task. Moreover, the overall shorter RT for the color stimuli (446 $\mathrm{ms})$ than for the shape stimuli $(477 \mathrm{~ms})$ after the practice implies that the incompatible mapping produced greater difficulty for responding to the shapes than to the colors. The relative lack of influence of the 100 trials of incompatible practice on the vertical Simon effect for the red-green color discriminations is in agreement with the results reported previously by $\mathrm{Vu}$ (2007). She found that 600 practice trials with the incompatible spatial mapping were required for the Simon effect to be a nonsignificant $-1 \mathrm{~ms}$.

We thought that any difference in results for the color and shape stimuli could be due to properties of the Simon effect across the RT distribution, possibly as a byproduct of differences in overall RT. As noted above, after the incompatible practice, RT was shorter with the color stimuli than with the shape stimuli. However, the bin analysis for Experiment 2 did not show a systematic effect of stimulus type or prior practice on the distribution functions. Thus, the relative lack of influence of the prior incompatible practice on the color discriminations compared to the shape discriminations apparently cannot be attributed to the distribution of Simon effects across the RT bins.

The bin analysis for Experiment 1 was even clearer in showing that, for the shape stimuli, the prior incompatible practice primarily had an effect across the entire RT distribution. This effect did not depend on whether the responses were made with the thumbs on the hand-held keypad or the index fingers with the keypad on the table. The results for responding on the keyboard in Experiment 2 were less clear but, as indicated, showed no significant interaction with bin. Conde et al. (2017) also reported not finding an interaction with bin for conditions with no practice, prior compatiblemapping practice, or prior incompatible-mapping practice in the shape discrimination task with their response device, with the exception that their data showed the Simon effect across conditions to decrease from $29 \mathrm{~ms}$ at bin 1 and to $-1 \mathrm{~ms}$ at bin 5.

Conde et al. (2017) conjectured that the more strongly decreasing vertical Simon effect in their study compared to others was due to the relation between the stimulus positions and responses being more direct in their study, for which the switches were placed one above the other in the frontal plane. We evaluated whether orientation in the frontal or transverse plane is a critical factor by performing bin analyses of the Simon effect for Zhong et al.'s (2018) Experiments 1 (no prior practice) and 3 (incompatible practice). The methods for those experiments were similar to the shape condition of the present Experiment 1, except with the thumb-operated number pad held in the frontal or transverse plane. The analyses showed a larger Simon effect without the incompatible practice (25 $\mathrm{ms}$ ) than with the practice ( $2 \mathrm{~ms})$, but unlike the present experiments and that of Conde et al. (2017), practice also interacted with bin. However, both functions were relatively flat across the first four RT bins, averaging $23 \mathrm{~ms}$ and $5 \mathrm{~ms}$ for the practice and no-practice conditions, respectively, and parted only at the last bin, being $37 \mathrm{~ms}$ for the no-practice condition and $-11 \mathrm{~ms}$ for the incompatible practice condition. Thus, in Zhong et al.'s (2018) experiments, the influence of the incompatible spatial mapping also was evident across the entire RT distribution, although its influence was largest for the slowest responses.

With regard to Conde et al.'s (2017) conjecture, the crucial three-way interaction of practice, bin, and number pad orientation showed an $F<1.0$ in Zhong et al.'s (2018) study. The absence of three-way interaction implies that whether the numeric pad was oriented vertically versus horizontally in their study did not matter much, if at all, suggesting that some factor other than vertical orientation of response keys in the frontal plane is necessary to yield consistently decreasing Simon effect functions. One possible factor is the amount of separation of the response keys, which is greater with Conde et al.'s (2017) response apparatus than with the numeric keypad. An alternative is that with Conde et al.'s apparatus, the response microswitch that is operated by a lever-pull with the left index finger is offset to the right, whereas the response microswitch that is operated by a lever-pull with the right index finger is offset to the left. Thus, their responses differ along the horizontal dimension as well as the vertical dimension. Proctor, Vu, and Nicoletti (2003) showed that when both stimuli and responses varied on horizontal and vertical dimensions, the Simon effect for the horizontal dimension showed the decreasing function across the RT distribution, whereas the vertical dimension did not. This result is consistent with the possibility that the decreasing functions obtained by Conde et al. (2017) may be a consequence of participants coding the response locations as left and right, as well as top and bottom.

The approximately additive influence of prior incompatible practice on the Simon effect across most of the RT distribution has also been found for the Simon effect with left and right locations. Proctor, Yamaguchi, Zhang, and Vu's (2009) Experiment 1 included conditions in which participants performed a left-right Simon task with relevant color discrimination after 84 trials of practice with an incompatible mapping of physical locations, arrow directions, or location words to the keypress responses. The Simon effect was uninfluenced by the prior practice with location words but eliminated after practice with the physical locations or arrow directions. Despite these differences in overall Simon effect size, the functions for all three of the transfer conditions were approximately parallel with that for the Simon effect with no prior practice. Proctor et al.'s (p. 444) interpretation, which is applicable to the vertical Simon effect as well, was that this result 
pattern suggests that the automatic-activation process that produces the Simon effect is intact, with practice (of an incompatible mapping) introducing a process whose time course is constant over the RT distribution and which counteracts the activation of the corresponding response that produces the Simon effect (De Jong, Liang, \& Lauber, 1994; Zhang \& Kornblum, 1997).

We can only conjecture as to why the Simon effect after prior practice with an incompatible mapping for 100 trials or less tends still to be significant for the vertical dimension but not for the horizontal dimension. When stimuli and responses varied in left and right locations, rather than top and bottom locations, $\mathrm{Vu}$ (2007) found a 19-ms Simon effect for a baseline condition in which the relevant stimulus dimension was the red and green color of a circle stimulus, and Proctor et al. (2009) found a 17-ms effect. Similarly, Proctor and Lu (1999) found a 20-ms Simon effect in their baseline condition when the relevant stimulus dimension was the letters $\mathrm{S}$ and $\mathrm{H}$. For vertically arrayed stimuli and responses, $\mathrm{Vu}$ (2007) found a 30-ms Simon effect with red and green stimuli, and Zhong et al. (2018) found a 26-ms Simon effect with circle and square stimuli, which is the same as the mean effect without practice in the present study. Across experiments, the baseline vertical Simon effect seems to average at least $6 \mathrm{~ms}$ larger than the baseline horizontal Simon effect. This difference may account in part for why the residual Simon effect after prior practice with an incompatible mapping for 100 trials or less is evident for the vertical dimension.

Based on the findings of the present study and the prior ones, we conclude that the plane along which the response keys are arrayed and the effectors used to execute the responses are not critical factors in producing the differences in vertical Simon effects after 100 practice trials with an incompatible spatial mapping. Thus, it is likely that the redgreen color discrimination used by $\mathrm{Vu}$ (2007) and circlesquare shape discrimination used by Conde et al. (2015, 2017) and Zhong et al. (2018) is the source of the discrepancy in results. There are several possible factors that could contribute to the difference in transfer of the incompatible mapping to the color discrimination task compared to the shape discrimination task, which we describe briefly below. However, we want to make clear that these factors are speculative at this point, and we are unable to directly attribute our findings to any one of them.

One possibility is that the red-green color discriminations and circle-square shape discriminations reflect different contributions of the ventral and dorsal visual streams. But, research seems to indicate that both streams are involved in the processing of color and shape (e.g., Claeys et al., 2004; Perry \& Fallah, 2014). Another possibility is that because the shape stimuli were equated for luminance but the color stimuli were not (which can affect RT; Fumarola et al., 2014), the color task could have been influenced by other types of congruency effects (e.g., bright with up and dim with down). However, it is not apparent why any congruency effect due to luminance would make the color stimuli less susceptible to an influence of the prior incompatible mapping.

The different result patterns for the color and shape versions of the Simon task in this study may also be a consequence of the specific stimuli used for the incompatible spatial task and transfer task. For example, because the visual system relies on sharp luminance changes to define the boundaries of objects (Vergeer, Anstis, \& van Lier, 2015), the association formed during the incompatible practice may be more strongly related to shape and therefore transfer more readily to shape stimuli. Also, because the color of the stimulus changes between red and green for the color discrimination task, this salient trial-to-trial feature change during the transfer session may make the stimuli more perceptually distinct from the neutral color stimuli in the practice session, reducing transfer of the incompatible S-R association. Whether the difference in result patterns for vertical red and green color stimuli and circle and square shape stimuli generalizes to other colors and shapes is an open question to which the answer will be informative as to the nature of the transfer effect.

Open Practices Statement The data and materials for all experiments are available from the authors upon request; none of the experiments were preregistered.

\section{References}

Cho, Y. S., \& Proctor, R. W. (2002). Influences of hand posture and hand position on compatibility effects for up-down stimuli mapped to leftright responses: Evidence for a hand-referent hypothesis. Perception \& Psychophysics, 64, 1301-1315.

Claeys, K. G., Dupont, P., Cornette, L., Sunaert, S., Van Hecke, P., De Schutter, E., \& Orban, G. A. (2004). Color discrimination involves ventral and dorsal stream visual areas. Cerebral Cortex, 14, 803822.

Conde, E. F., Fraga-Filho, R. S., Lameira, A. P., Mograbi, D. C., Riggio, L., \& Gawryszewski, L. G. (2015). Influence of short incompatible practice on the Simon effect: transfer along the vertical dimension and across vertical and horizontal dimensions. Experimental Brain Research, 233, 3313-3321.

Conde, E. F. Q., Fraga Filho, R. S., Lameira, A. P., Riggio, L., \& Gawryszewski, L. G. (2017). Stimulus-response dimensional overlap elicits a decreasing Simon effect along the vertical dimension. Estudos de Psicologia, 22, 358-365.

De Jong, R., Liang, C.-C., \& Lauber, E. (1994). Conditional and unconditional automaticity: A dual-process model of effects of spatial stimulus-response correspondence. Journal of Experimental Psychology: Human Perception and Performance, 20, 731-750.

Fumarola, A., Prpic, V., Da Pos, O., Murgia, M., Umiltà, C., \& Agostini, T. (2014). Automatic spatial association for luminance. Attention, Perception, \& Psychophysics, 76, 759-765.

Lu, C. H., \& Proctor, R. W. (1995). The influence of irrelevant location information on performance: A review of the Simon and spatial Stroop effects. Psychonomic Bulletin \& Review, 2, 174-207. 
Perry, C. J., \& Fallah, M. (2014). Feature integration and object representations along the dorsal stream visual hierarchy. Frontiers in Computational Neuroscience, $8,84$.

Proctor, R. W., \& Lu, C. H. (1999). Processing irrelevant location information: Practice and transfer effects in choice-reaction tasks. Memory \& Cognition, 27, 63-77.

Proctor, R. W., Miles, J. D., \& Baroni, G. (2011). Reaction-time distribution analysis of spatial correspondence effects. Psychonomic Bulletin \& Review, 18, 242-266.

Proctor, R. W., \& Vu, K. P. L. (2006). Stimulus-response compatibility principles: Data, theory, and application. Boca Raton, FL: CRC Press.

Proctor, R. W., Vu, K.-P. L., \& Nicoletti, R. (2003). Does right-left prevalence occur for the Simon effect? Perception \& Psychophysics, 65, $1318-1329$

Proctor, R. W., Yamaguchi, M., Zhang, Y., \& Vu, K.-P. L. (2009). Influence of visual stimulus mode on transfer of acquired spatial associations. Journal of Experimental Psychology: Learning, Memory, and Cognition, 35, 434-445.

Simon, J. R. (1969). Reactions toward the source of stimulation. Journal of Experimental Psychology, 81, 174-176.

Simon, J. R. (1990). The effects of an irrelevant directional cue on human information processing. In R. W. Proctor \& T. G. Reeve (Eds.), Stimulus-response compatibility: An integrated perspective (pp. 31-86). Amsterdam: North-Holland.

Stürmer, B., Leuthold, H., Soetens, E., Schröter, H., \& Sommer, W. (2002). Control over location-based response activation in the Simon task: behavioral and electrophysiological evidence. Journal of Experimental Psychology: Human Perception and Performance, $28,1345$.

Tagliabue, M., Zorzi, M., Umiltà, C., \& Bassignani, F. (2000). The role of long-term-memory and short-term-memory links in the Simon effect. Journal of Experimental Psychology: Human Perception and Performance, 26, 648-670.

Töbel, L., Hübner, R., \& Stürmer, B. (2014). Suppression of irrelevant activation in the horizontal and vertical Simon task differs quantitatively not qualitatively. Acta Psychologica, 152, 47-55.
Vergeer, M., Anstis, S., \& van Lier, R. (2015). Flexible color perception depending on the shape and positioning of achromatic contours. Frontiers in Psychology, 6, 620.

$\mathrm{Vu}, \mathrm{K}$.P. L. (2007). Influences on the Simon effect of prior practice with spatially incompatible mappings: Transfer within and between horizontal and vertical dimensions. Memory \& Cognition, 35, 14631471.

Vu, K.-P. L., Pellicano, A., \& Proctor, R. W. (2005). No overall right-left prevalence for horizontal. Perception \& Psychophysics, 67, 929938.

Vu, K.-P. L., \& Proctor, R. W. (2001). Determinants of right-left and topbottom prevalence for two-dimensional spatial compatibility. Journal of Experimental Psychology: Human Perception and Performance, 27, 813-828.

Wang, H., \& Proctor, R. W. (1996). Stimulus-response compatibility as a function of stimulus code and response modality. Journal of Experimental Psychology: Human Perception and Performance, 22, 1201-1217.

Wobbrock, J. O., Myers, B. A., \& Aung, H. H. (2008). The performance of hand postures in front-and back-of-device interaction for mobile computing. International Journal of Human-Computer Studies, 66, $857-875$.

Zhang, J., \& Kornblum S. (1997). Distributional analysis and De Jong, Liang, and Lauber's (1994) dual-process model of the Simon effect. Journal of Experimental Psychology: Human Perception and Performance, 23, 1543-1551.

Zhong, Q., Xiong, A., Vu, K. P. L., \& Proctor, R. W. (2018). Vertically arrayed stimuli and responses: transfer of incompatible spatial mapping to Simon task occurs regardless of response-device orientation. Experimental Brain Research, 236, 175-185.

Publisher's note Springer Nature remains neutral with regard to jurisdictional claims in published maps and institutional affiliations. 